\title{
Von den Pflanzen angetrieben
}

Was hat sich in den letzten

3-4 Jahrzehnten geändert, in denen Sie sich mit dem Thema Phytotherapie beschäftigt haben?

Die Phytotherapie ist in diesen Jahrzehnten sicher bekannter geworden, nicht nur in der Öffentlichkeit, sondern vor allem auch in der Medizin. Wir haben grosse Fortschritte im Bereich der Forschung gemacht, sodass heute doch viel mehr klinische Studien und pharmakologische Daten vorhanden sind als vor 30 oder 40 Jahren. Es hat sich auch sonst viel bewegt, seitdem ich 1974 mit dem Start zu meiner Dissertation in den Fachbereich der Arzneipflanzenforschung eingestiegen bin. Die Schweizerische Medizinische Gesellschaft für Phytotherapie (SMGP) wurde 1988, also vor 26 Jahren, gegründet. Wenn man sich das damalige Fort- und Weiterbildungsangebot der SMGP anschaut, war vieles noch sehr qualitativ. Die Basis waren verschiedene Lehrbücher, in denen beschrieben wurde, welche Pflanze im Prinzip wie wirkt. Heute können wir im Rahmen unseres Fähigkeitsprogramms Phytotherapie bei sehr vielen Indikationen auf valide klinische Daten zurückgreifen. Ich denke, das ist ein erheblicher Fortschritt.

\section{Was sind die Treiber dieser Entwicklung gewesen?}

Im Wesentlichen haben die gestiegenen Anforderungen in der Schweiz und in Deutschland dafür gesorgt, dass es diesen Fortschritt gegeben hat. Man blieb in diesen Ländern beim Konzept, dass pflanzliche Zubereitungen, die eine Indikation beanspruchen, Arznei-
An der Zürcher Hochschule für Angewandte Wissenschaften (ZHAW) ist Professor Beat Meier als Dozent für Phytopharmazie am 1. September nach 40-jährigem Engagement für die Arzneipflanzenforschung in den Ruhestand getreten. Ein Gespräch über die Entwicklung der Phytotherapie in den letzten 40 Jahren und seine Pläne für die Zukunft.

mittel sein sollen. Das führte zu einem gewissen Druck, etwas zu tun, und in der Folge gab es verschiedene Firmen, die sich der klinischen Forschung auf diesem Gebiet verschrieben haben. Sie führten dann die nötigen Untersuchungen durch. Es gibt daher nur wenige anwendungsorientierte Studien, die ausschliesslich an Hochschulen gemacht wurden. Zusammenfassend kann man sagen, dass es die Anforderung und die Notwendigkeit waren, dass ein Arzneimittel auch eine gewisse Dokumentation braucht, die uns den Fortschritt der letzten Jahrzehnte gebracht haben.

\section{Also konnte die Phytotherapie mit eigentlich klassischen und schulmedizinischen Methoden «mithalten»?}

Auf alle Fälle in der Methodik, in der Menge der Untersuchungen natürlich nicht. Eine umfassende Forschungsaktivität wie in der klassischen Pharmaindustrie scheitert schon daran, dass die notwendigen Finanzen einfach nicht da sind. Es gibt keine Blockbuster in der Phytotherapie, sondern vor allem kleinere Präparate, die sehr oft lokal oder national geprägt sind. Es gibt schon internationale Trends, aber jedes Land behandelt die Arzneimittel aus Pflanzen anders.

Bei den Wirkstoffen aus Pflanzen hat jedes Land mehr oder weniger seine eigenen Richtlinien. Europa ist, was

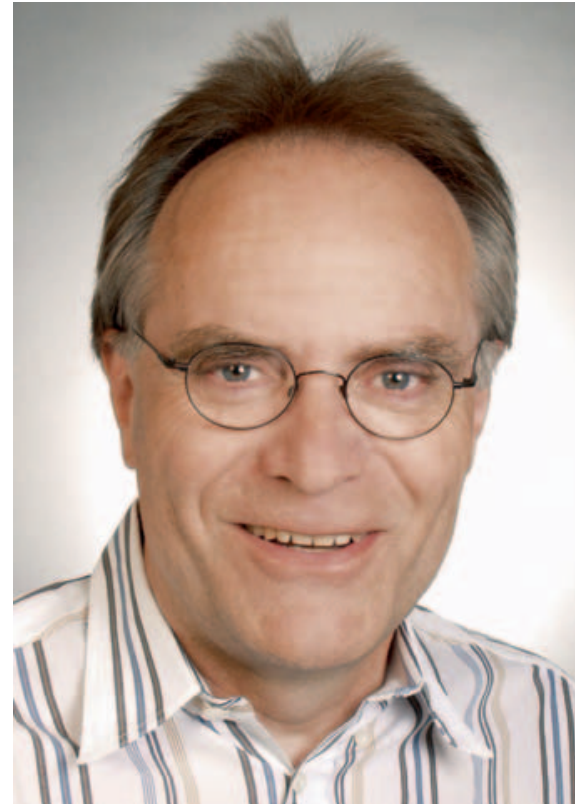

das betrifft, noch einigermassen homogen - aber wenn man auf andere Kontinente blickt, wird es sehr uneinheitlich. Auch die Traditionen sind ganz unterschiedlich. Das sorgt dafür, dass die Phytotherapie ganz andere Rahmenbedingungen hat als die Schulmedizin mit ihren Arzneimitteln.

\section{Was sind denn die grössten Unterschiede zwischen Ländern in Europa, was die Verwendung von Phytotherapeutika betrifft?}

In der Schweiz, in Deutschland und in Österreich blieben die Arzneipflanzen auch nach dem Aufkommen der 
synthetisch gewonnenen, in ihrer Struktur klar definierten Wirkstoffe eine Basis für Arzneimittel. In den meisten anderen Ländern, wie etwa in den USA sowie in Frankreich, Skandinavien, Italien oder Spanien, ist dieses Konzept nach dem Zweiten Weltkrieg weitgehend aufgegeben worden. In Skandinavien oder England gibt es zwar Zulassungen, doch sie sind alle älteren Datums und passen nur noch selten in ein modernes Konzept. Wirklich verankert und auch von der Industrie getragen sind pflanzliche Arzneimittel eigentlich nur in Deutschland und in der Schweiz.

\section{Worin liegen diese Kulturunter- schiede begründet, dass gerade im deutschsprachigen Raum die Heilpflanzen als Arzneimittel gelten?}

Das ist für mich schwierig zu sagen. Ich vermute, das hat einerseits mit der Tradition zu tun, die deutlich wird, wenn man die Spur der traditionellen Arzneipflanzenbücher zurückverfolgt. Diese kommen in der Regel aus dem deutschsprachigen Raum. Das zeigt, dass es eigentlich seit dem Mittelalter im deutschsprachigen Raum eine entsprechende Tradition gibt. Andererseits hat das mit der Politik zu tun. In England oder Skandinavien hat man $\mathrm{ab}$ einem gewissen Zeitpunkt nur noch Wirkstoffe als Arzneimittel betrachtet, die chemisch einheitlich definiert werden konnten. Alles andere wurde an den Rand gedrängt. Es würde sich wohl lohnen, die Ursachen dieser Unterschiede in den Traditionen einmal historisch aufzuarbeiten.

Sehen Sie eine Tendenz, dass sich pflanzliche Arzneimittel auch in den anderen Ländern mehr oder weniger einen festen Platz erobern?

Einen Platz haben sie fast überall, aber oft nicht als Arzneimittel. Den- ken Sie an die Entwicklung in den USA in den letzten 20 Jahren. Dort ist ihre Verbreitung enorm - die "Herbals» sind jedoch keine von der Food and Drug Administration (FDA) zugelassenen Arzneimittel. Verschiedene Probleme mit der Qualität und mit Verunreinigungen haben dazu geführt, dass die Herbals in den USA nun auch nach GMP(Good Manufacturing Practice)-Richtlinien hergestellt werden müssen und die Aussagen zur Wirksamkeit stark limitiert worden sind. Die FDA hat sich da stark engagiert. In Europa gibt es immer mehr sogenannte Nahrungsergänzungsmittel mit Arzneipflanzen. Da ist es oft, auch für den Fachmann, schwierig zu unterscheiden, welche Produkte hohen Ansprüchen gerecht werden und welche möglichst billig produziert wurden und eigentlich nur den Namen einer Pflanze tragen. Klinische Forschung ist da kein grosses Thema.

\section{Eine strikte Regulierung käme den pflanzlichen Arzneimitteln entgegen, weil dadurch die Qualitätsstandards verbessert würden?}

Das käme der Phytotherapie als medizinischer Disziplin entgegen. Ob sie für deren Verbreitung ein Vorteil wäre, ist eine andere Frage. Ich würde es begrüssen, wenn die Phytotherapeutika in allen Ländern Arzneimittel wären.

\section{Was ist für Sie auf der Seite der Forschung die überraschendste Entdeckung in der Phytotherapie in den letzten Jahrzehnten gewesen?}

In der phytotherapeutischen Forschung wird im Wesentlichen die Tradition belegt. Entsprechend erhöhen sich der Wissensstand und die Sicherheit des Wissens. Die meisten pflanzlichen Arzneimittel kennt man schon lange, und sie sind teilweise schon seit Jahrhunderten in diversen Büchern be-

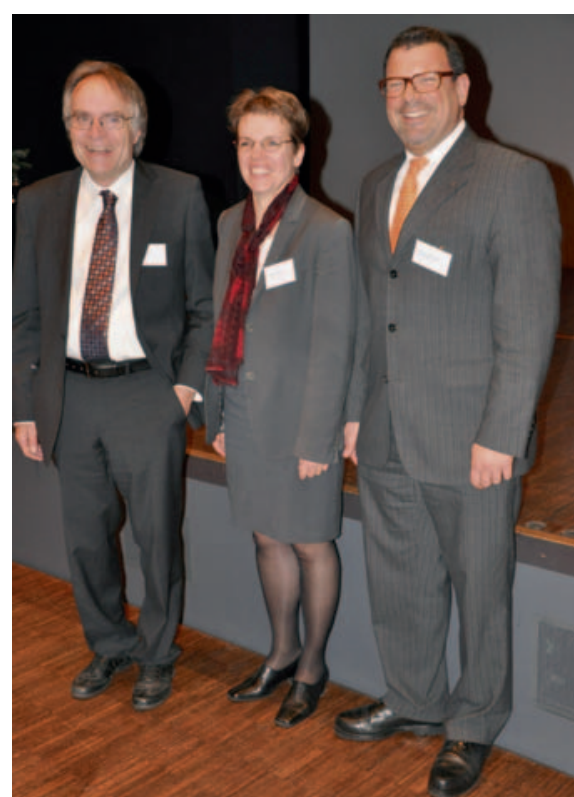

Abb. Drei der treibenden Kräfte in der SMGP (von links nach rechts): Geschäftsführer Prof. Dr. Beat Meier, Vizepräsidentin Dr. Beatrix Falch und Präsident Dr. Roger Eltbogen.

schrieben. Immerhin durfte ich bei der Entdeckung der antiallergischen Wirksamkeit der Pestwurz mit dabei sein und das entsprechende Präparat mitentwickeln. Und bei der Entwicklung von Johanniskraut zu einem modernen Arzneimittel zur Behandlung von Verstimmungszuständen und depressiven Beschwerden war ich auch dabei. Da zeigt sich auch, wie Arzneimittel zeitabhängig an Bedeutung gewinnen können. Vor 1990 war Johanniskraut eine nicht sehr bedeutende Arzneipflanze. Erst in den letzten 20 Jahren sind die damit zu behandelnden Beschwerden in der Wohlstandsgesellschaft zu einem Problem geworden, und Johanniskraut erlebte einen Wandel zur heute wohl bekanntesten und am besten dokumentierten Arzneipflanze.

Bei den synthetischen Arzneimitteln gibt es die ständige Suche nach neuen Molekülen und nach der Verbesserung von Wirkstoffen. Es geht darum, etwas Bestehendes noch besser zu machen. Da ist die medizinische Phytotherapie auf einem anderen Weg? 
Sie versucht wissenschaftlich zu belegen, was man schon kennt. Natürlich, es gibt auch eine namhafte Forschung, die versucht, neue Substanzen mit Wirkungspotenzial in den Pflanzen zu finden. Daraus entstehen teilweise ja auch Arzneimittel. Es ist ja immer noch so, dass etwa die Hälfte der neuen Wirkstoffe bei den synthetischen Arzneimitteln ihr Modell in der Natur haben. Es passiert aber sehr selten, dass man ein neues Wirksamkeitsprofil für eine seit längerer Zeit bekannte Arzneipflanze bis hin zum Arzneimittel entwickelt, so wie das bei der Pestwurz der Fall war.

\section{Gibt es Vorteile von pflanzlichen Arzneimitteln gegenüber synthetischen Wirkstoffen?}

Bei pflanzlichen Arzneimitteln haben wir ein Vielstoffgemisch vor uns, das eine pleiotrope Wirkung entfaltet. Man muss sich vor Augen halten, dass die Physiologie des Menschen sehr komplex ist. Mit sogenannten Monodrugs wird versucht, punktuell einzugreifen. Entsprechend ist die Wirkung weniger ausbalanciert, als wenn man das mit pflanzlichen Mitteln versucht. Pflanzliche Arzneimittel werden ja meistens bei recht komplexen gesundheitlichen Situationen, wie etwa bei rheumatischen Beschwerden, bei Magen-Darm-Problemen, beim prämenstruellen Syndrom oder bei Beschwerden in der Menopause, verwendet. Das sind alles sehr komplexe Beschwerden, bei denen die pflanzlichen Arzneimittel multifaktoriell eingreifen, regulierend wirken und ein weitgehend inexistentes Nebenwirkungsprofil haben.

\section{Wo steht die SMGP, deren Geschäftsleiter Sie vorläufig bleiben, heute?}

Die SMGP ist als Gesellschaft gut aufgestellt. Wir haben unser Fähigkeitsprogramm, das mittlerweile breit anerkannt ist. Vor 3 Jahren hat es die Anerkennung durch das Schweizerische Institut für ärztliche Weiter- und Fortbildung (SIWF) und damit der FMH endlich gefunden. Es ist zuvor schon von den Schweizer Verbänden der Veterinärmediziner und der Apotheker qualifiziert worden. Das Programm zur Aus- und Weiterbildung wird ständig an die neuen Erkenntnisse angepasst. Das Ziel der SMGP bleibt konstant: Wir wollen der Phytotherapie als nützlicher Ergänzung in der modernen Medizin zu ihrem Platz verhelfen. Das ist und bleibt der Leitgedanke.

\section{Wie ist die Begeisterung von jüngeren Ärzten für die Phytotherapie?}

Das ist schwierig zu sagen. Das Studium ist ausgefüllt, und in den ersten Berufsjahren haben die jungen Ärzte oft so viel zu tun, dass sie sich selten mit zusätzlichen Aspekten der Medizin beschäftigen können. So bleibt das Interesse punktuell. Das Interesse erwacht meist erst in der Praxis im Kontakt mit den Patienten. Auf alle Fälle braucht es eine Affinität zu den Pflanzen: Man muss sie gernhaben und eine gewisse Verbundenheit mit der Natur besitzen, um überhaupt in dieses Gebiet einzusteigen.

\section{Wie sieht die Akzeptanz der Phytotherapie vonseiten der niedergelassenen Ärzte aus?}

Die hat sich verbessert. Wir haben in der Spezialitätenliste bald 100

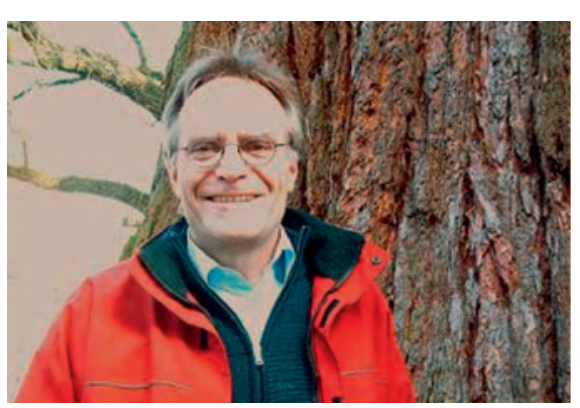

pflanzliche Arzneimittel, die verschrieben werden können und von der Grundversicherung vergütet werden müssen. Das erhöhte die Akzeptanz. Wir haben aber nach wie vor nur eine kleine Gruppe von Hausärzten, die den Nutzen der Phytotherapie für den Patienten erfasst haben.

\section{Wie werden Sie nach Ihrer Pensionierung Ihre Zeit verbringen?}

Ich bin bis auf Weiteres an 2 Tagen im Monat an der ZHAW anzutreffen. Hier hat Frau Evelyn Wolfram meine Nachfolge in der Fachgruppe Phytopharmazie übernommen. Ich möchte ihr helfen, den längst eingeleiteten Übergang weiterhin fliessend zu gestalten, und sie darin unterstützen, dass der Support von extern weiterhin gewährleistet ist. Als Fachhochschule hat die ZHAW ja nur eine beschränkte eigene Forschungsförderung, sodass für alle Forschungsarbeiten Drittmittel generiert werden müssen. Ich bin zudem weiterhin mit der Pharmakopöe beschäftigt und als Präsident von zwei Fachausschüssen, die schwerpunktmässig mit Arzneipflanzen zu tun haben, aktiv. Die SMGP-Geschäftsstelle betreue ich weiterhin; eine Übergabe während der nächsten Jahre ist geplant. Daneben geniesse ich die Freiheiten, vermehrt in der Natur zu sein, zusammen mit meiner Frau und unseren beiden Hunden, und mit vermehrtem Training meine physische Verfassung auf angemessenem Level zu halten.

Ich darf sagen, dass ich immer gerne gearbeitet habe. Ich hatte die Gnade, dass ich in meinem Fachgebiet Arbeitsstellen fand, die mich faszinierten. Ich konnte verschiedenste Facetten der Arzneipflanzen untersuchen. So blicke ich auf eine berufliche Laufbahn zurück, in der ich nichts Negatives erlebt habe. Ich wurde von den Pflanzen angetrieben.

Interview: Oliver Klaffke 